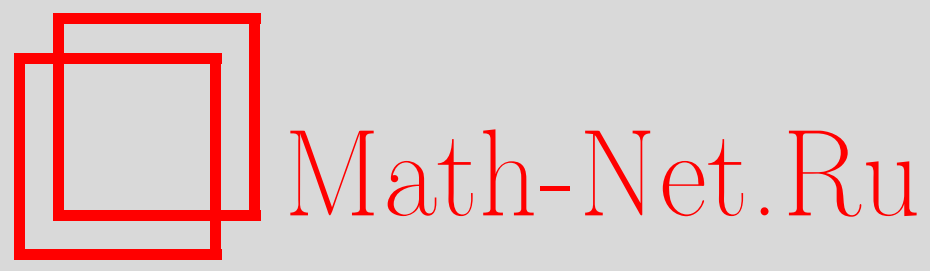

В. П. Гурарий, Д. У. Х. Гиллам, О функциях, однозначно определяемых своими асимптотическими разложениями, Функи. анализ и его прил., 2006, том 40, выпуск 4, 33-48

DOI: https://doi.org/10.4213/faa852

Использование Общероссийского математического портала MathNet.Ru подразумевает, что вы прочитали и согласны с пользовательским соглашением http://www . mathnet.ru/rus/agreement

Параметры загрузки:

IP : 54.92 .164 .108

26 апреля 2023 г., 02:20:31

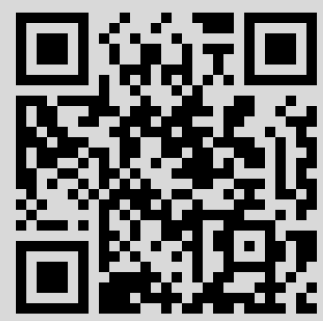




\title{
О функциях, однозначно определяемых своими асимптотическими разложениями
}

\author{
(c) 2006. Д. У. Х. ГилЛАм, В. П. ГУрарий
}

Памлти Бориса Яковлевича Левина

\section{§1. Введение}

В 1912 г. Дж. Ватсон опубликовал следующий результат [17, Sec. 8, Theorem V]. ТеОРема ЕДИНСТВенНостИ ВАТСОНА. Предположим, что последовательность комплексных чисел $\left\{p_{0}, p_{1}, \ldots\right\}$ и функиия $P(z)$ удовлетворяют следующим условиям:

(i) $P(z)$ является аналитической и однозначной функиией в секторе $S(\alpha, \beta)$;

(ii) для всех $z \in S(\alpha, \beta)$ и для каждого $n, n=0,1, \ldots$,

$$
\left|\mathscr{R}_{n, P}(z)\right| \leqslant M \frac{n !}{a^{n}|z|^{n+1}},
$$

где положительные постоянные $M$ и а не зависят от $z$ и $n$, но могут зависеть от $P(z)$.

Если раствор сектора $S(\alpha, \beta)$ удовлетворяет неравенству $\beta-\alpha>\pi$, то функиия $P(z)$ однозначно определяется условиями (i) и (ii): две функции $P_{1}(z)$ и $P_{2}(z)$, для которых (i) и (ii) выполняются с одной и той же последовательностъю $\left\{p_{0}, p_{1}, \ldots\right\}$, тождественны в $S(\alpha, \beta)$.

Здесь $S(\alpha, \beta)$ обозначает сектор $\{z \in \mathbb{C}: 0<|z|<\infty, \alpha<\arg z<\beta\}$, а число $\beta-\alpha$ называется раствором этого сектора. Для заданных функции $P(z)$, аналитической в $S(\alpha, \beta)$, и последовательности комплексных чисел $\left\{p_{0}, p_{1}, \ldots\right\}$ выражение

$$
\mathscr{R}_{n, P}(z)=P(z)-\sum_{k=0}^{n-1} \frac{p_{k}}{z^{k+1}}
$$

называется остатком.

Поскольку множество функций, удовлетворяющих условиям (i) и (ii), линейно, теорему Ватсона достаточно доказать для случая, когда последовательность $\left\{p_{0}, p_{1}, \ldots\right\}$ состоит из нулей. В таком случае

$$
|P(z)| \leqslant M \frac{n !}{a^{n}|z|^{n+1}}, \quad n=0,1, \ldots, z \in S(\alpha, \beta) .
$$

Фиксируя $z,|z|>1 / a$, и минимизируя по $n$ правую часть неравенств (2), получаем

$$
|P(z)| \leqslant M_{a} e^{-a|z|},
$$


где $M_{a}=4 M \sqrt{2 \pi} a$. Для завершения доказательства, установления импликации $(3) \Longrightarrow P(z) \equiv 0$, нам понадобится следующая лемма.

ЛЕмма 1. Предположим, что функиия $P(z)$, аналитическая в секторе $S(\alpha, \beta)$ раствора, большего $\pi$, удовлетворяет в этом секторе неравенству $(3)$. Тогда $P(z) \equiv 0$.

Доказательство этой леммы, опирающееся на принцип Фрагмена-Линделёфа, приведено в [8, Sec. 8.11].

В том случае, когда раствор сектора $S(\alpha, \beta)$ удовлетворяет противоположному неравенству $\beta-\alpha<\pi$, теорема единственности, бесспорно, неверна. В самом деле, задав $\delta, 0<\delta<\pi / 2$, введем в рассмотрение функцию $P(z)$ вида $P(z)=\varphi(z) e^{-z} / z$, где $\varphi(z)$ - аналитическая и ограниченная функция в секторе $S=S(-\pi / 2+\delta, \pi / 2-\delta)$. Как показывают элементарные неравенства

$$
e^{-|z|}<\frac{n !}{|z|^{n}}, \quad n=0,1, \ldots,
$$

для каждой такой функции условие (1) выполняется, если положить $M=$ $\sup _{S}|\varphi(z)|, a=\sin \delta$ и $p_{k}=0$ для $k=0,1, \ldots$

Таким образом, множество функций, удовлетворяющих условиям (i) и (ii) теоремы Ватсона с $\left\{p_{0}, p_{1}, \ldots\right\}=\{0,0, \ldots\}$, оказывается довольно богатым.

Сопоставление двух противоположных случаев, $\beta-\alpha>\pi$ и $\beta-\alpha<\pi$, дает основание рассматривать случай $\beta-\alpha=\pi$ как критический случай, а значение $\pi$ - как критическое значение раствора $\beta-\alpha$.

В действительности утверждение теоремы единственности Ватсона остается верным и в критическом случае. Несмотря на то что доказательство, приведенное в [8, Sec. 8.11], неприменимо к этому случаю, все же остается возможность использовать теорему Фрагмена-Линделёфа для получения единственности, и доказательство может быть сделано даже более простым. Предположим, что неравенство (3) справедливо в секторе $S(-\pi / 2, \pi / 2)$. Задавая $b, b>0$, рассмотрим в секторе $S(-\operatorname{arctg}(b / a), \operatorname{arctg}(b / a))$ функцию вида $P_{b}(z)=P(z) e^{b z}$. Так как на границе этого сектора $a|\operatorname{Im} z|-b \operatorname{Re} z=0$, то на этой границе выполняется неравенство $\left|P_{b}(z)\right| \leqslant M_{a}$. Применение стандартной теоремы ФрагменаЛинделёфа ведет к такой же оценке и внутри сектора. Поэтому для $z>0$ получается оценка $|P(z)| \leqslant M_{a} e^{-b z}$. Учитывая, что $b$ не зависит от $z$, мы устремляем $b$ к $+\infty$ и получаем $P(z) \equiv 0$.

\section{§2. Разложение Жевре порядка $k$}

Теорема единственности Ватсона может быть переформулирована для любого сектора $S(\alpha, \beta)$ на римановой поверхности $\log z$ раствора, большего или равного $\pi / k$, где $k$ - положительное число. В таком случае условие (ii) должно быть заменено следующим условием:

$$
\left|\mathscr{R}_{n, P}(z)\right| \leqslant M \frac{(n !)^{1 / k}}{(k a|z|)^{n+1}}, \quad n=0,1, \ldots .
$$

Если раствор сектора $S(\alpha, \beta)$ удовлетворяет неравенству $\beta-\alpha \geqslant \pi / k$, то функция $P(z)$ однозначно определяется последовательностью коэффициентов $\left\{p_{0}, p_{1}, \ldots\right\}$. 
Этот результат получается следующим образом. Из оценок (4) вместо (3) выводится неравенство $|P(z)| \leqslant M_{a} e^{-a|z|^{k}}$, которое приводится к виду (3) с помощью отображения $\zeta=z^{k}$.

Сопоставляя снова два противоположных случая $\beta-\alpha>\pi / k$ и $\beta-\alpha<\pi / k$, мы называем сектор $S(\alpha, \beta)$ критическим, если его раствор равен $\pi / k$.

Теория Ватсона, основанная на оценках (1), получила дальнейшее развитие в работе Жевре 1918 г. [5], который применил ее в теории дифференциальных уравнений в частных производных, в частности, для уравнения теплопроводности, и работе Ф. Неванлинны 1919 г. [9], результаты которого будут обсуждаться ниже.

Карлеман, см. [3], распространил результат теоремы единственности Ватсона на более общую ситуацию. Он заменил $n$ ! в оценках (1) последовательностью положительных чисел $m_{n}$ и, предполагая некоторую регулярность роста чисел $m_{n}$, когда $n \rightarrow \infty$, указал необходимые и достаточные условия единственности.

Разложения, удовлетворяющие условиям, подобным (4), получили название разложений Жевре порядка $k$ (см. [5], [11] и [10]).

С начала прошлого века было известно, что в асимптотической теории систем линейных дифференциальных уравнений, имеющих в некоторой точке комплексной плоскости иррегулярную сингулярность ранга Пуанкаре $k$, особое значение имеют секторы раствора $\pi / k$. Сравнительно недавно было обнаружено, что асимптотические разложения Пуанкаре, ассоциированные с регулярными решениями таких систем, в действительности являются разложениями Жевре (см. [10]). Это свойство асимптотических разложений для регулярных решений оказалось справедливым не только для линейных систем, но и для существенно более широкого класса аналитических дифференциальных уравнений. Такое достижение в теории аналитических дифференциальных уравнений основано на следующем определении

ОПРЕДЕЛЕНИЕ. Рассмотрим в $z$-плоскости некоторый сектор $S(\alpha, \beta)$, и пусть $P(z)$ - однозначная аналитическая функция в этом секторе. Введем в рассмотрение некоторый формальный степенной ряд с комплексными коэффициентами вида $\sum_{j=0}^{\infty} p_{j} / z^{j+1}$. Если для каждого собственного подсектора $S^{\prime}$ сектора $S(\alpha, \beta), \overline{S^{\prime}} \subset S(\alpha, \beta)$, можно указать положительные постоянные $M\left(S^{\prime}\right)$ и $a\left(S^{\prime}\right)$, такие, что

$$
\left|\mathscr{R}_{n, P}(z)\right| \leqslant M\left(S^{\prime}\right) \frac{(n !)^{1 / k}}{\left(k a\left(S^{\prime}\right)\right)^{n}|z|^{n+1}}, \quad z \in S^{\prime},
$$

то этот формальный ряд называется разложением Жевре порядка $k, k>0$, функиии $P(z)$ в секторе $S(\alpha, \beta)$.

\section{§3. Основная теорема}

Мы развиваем результат Ватсона в следующем направлении. Мы рассматриваем критический сектор $S$ раствора, равного $\pi$, и выясняем, каким условиям должны подчиняться $M\left(S^{\prime}\right)$ и $a\left(S^{\prime}\right)$ для того, чтобы функция $P(z)$, обладающая в этом секторе разложением Жевре указанного выше вида, однозначно определялась коэффициентами этого разложения. Предположим для простоты, 
что $k=1$ и $S=S(-\pi / 2, \pi / 2)$. Поскольку наиболее существенным параметром, определяющим положение подсектора $S^{\prime}$ внутри $S$, является угловое отклонение границы этого подсектора от границы сектора $S$, нам достаточно ограничиться рассмотрением подсекторов $S^{\prime}$ вида $S^{\prime}=S(-\pi / 2+\delta, \pi / 2-\delta)$, где $0<\delta<\pi / 2$. В таком случае вместо функций $M\left(S^{\prime}\right)$ и $a\left(S^{\prime}\right)$ мы будем рассматривать $M(\delta)$ и $a(\delta)$, предполагая без ограничения общности, что $\log M(\delta)>1$.

Для критического сектора $S(-\pi / 2, \pi / 2)$ справедливо следующее обобщение теоремы единственности Ватсона.

Теорема 1. Предположим, что в секторе $S(-\pi / 2, \pi / 2)$ функиия $P(z)$ допускает разложсене Жевре порядка 1 . Если $M(\delta)$ и а $(\delta)$ удовлетворяют условиям

$$
\int_{0}^{\pi / 2} \log \log M(\delta) d \delta<\infty
$$

$u$

$$
\frac{\delta}{a(\delta)} \rightarrow 0 \quad \text { nрu } \delta \rightarrow 0
$$

то $P(z)$ однозначно определяется коэффициентами своего разложения Жевре.

Можно показать, что нарушение одного из условий (6) или (7) ведет к потере единственности.

ЗАмечАние 1 . В тех ситуациях, когда функция $P(z)$ и формальный степенной ряд $\widehat{P}(z)=\sum_{j=0}^{\infty} p_{j} / z^{j+1}$ являются решениями, регулярным и формальным соответственно, одного и того же аналитического дифференциального уравнения, случаи

$$
M(\delta)=M \exp \left(b / \delta^{\gamma}\right)
$$

и

$$
M(\delta)=M \exp (b / \delta),
$$

где положительные числа $M, b$ и $\gamma$ не зависят от $\delta$, представляют особый интерес.

\section{§4. Формула Стирлинга}

Аналитические функции, удовлетворяющие условиям (1), (4) или (5), встречались в анализе с давних пор.

Мы напомним пример, основанный на формуле Стирлинга для гамма-функции Эйлера $\Gamma(z)$, аналитической всюду в комплексной области за исключением точек $z=0,-1, \ldots$ отрицательной полуоси, где она имеет простые полюсы. В секторе $S(-\pi / 2, \pi / 2)$ для $\Gamma(z)$ имеет место интегральное представление

$$
\Gamma(z)=\int_{0}^{\infty} t^{z-1} e^{-t} d t .
$$

Формула Стирлинга означает, что

$$
\ln \Gamma(z)=\left(z-\frac{1}{2}\right) \ln z-z+\frac{1}{2} \ln 2 \pi+o(1), \quad z \rightarrow+\infty .
$$


Введем в рассмотрение так называемую функиию Бине

$$
P(z)=\ln \Gamma(z)-\left(z-\frac{1}{2}\right) \ln z+z-\frac{1}{2} \ln 2 \pi
$$

и сопоставим этой функции последовательность $\left\{p_{0}, p_{1}, \ldots\right\}$ таким образом, чтобы при некотором выборе $M$ и $а$ выполнялись соотношения (1).

Согласно первой формуле Бине, имеет место представление

$$
P(z)=\int_{0}^{\infty} e^{-z t} F(t) d t
$$

где

$$
F(t)=\frac{1}{t}\left(\frac{1}{2}-\frac{1}{t}+\frac{1}{e^{t}-1}\right) .
$$

Поскольку функция $F(t)$ голоморфна внутри круга $|t|<2 \pi$, она может быть представлена в нем своим рядом Тейлора $\sum_{k=0}^{\infty} f_{k} t^{k}$. Подставляя в формулу (11) вместо функции $F(t)$ ее ряд Тейлора и меняя формально порядки суммирования и интегрирования, мы получаем

$$
\widehat{P}(z)=\sum_{k=0}^{\infty} \frac{p_{k}}{z^{k+1}}, \quad p_{k}=f_{k} k ! .
$$

Пользуясь тем, что функция $F(t)$ четна, $F(-t)=F(t)$, нетрудно заключить, что $p_{2 k-1}=0$ для $k=1,2, \ldots$ Более детальный анализ позволяет указать точное выражение для четных коэффициентов

$$
p_{2 k-2}=\frac{B_{2 k}}{2 k(2 k-1)}, \quad k=1,2, \ldots .
$$

Здесь $B_{2 k}, k=0,1, \ldots$, являются числами Бернулли, которые могут быть определены как коэффициенты ряда Тейлора

$$
\frac{t}{e^{t}-1}=1-\frac{t}{2}+\sum_{k=2}^{\infty} B_{2 k} \frac{t^{2 k}}{(2 k) !}
$$

см., например, формулы 23.1.1-23.1.3 и 6.1.42 в [1]. Таким образом, формальный ряд $\widehat{P}(z)$ может быть записан в виде

$$
\widehat{P}(z)=\sum_{k=1}^{\infty} \frac{B_{2 k}}{2 k(2 k-1) z^{2 k-1}},
$$

и в таком виде он известен как ряд Стирлинга ${ }^{1)}$.

Формула (11) дает возможность показать, что $\widehat{P}(z)$ является разложением Жевре функции $P(z)$ в секторе $S(-\pi / 2, \pi / 2)$. Действительно, при $z \in S(-\pi / 2$, $\pi / 2)$ имеют место следующие неравенства:

1) Похожее выражение для ряда Стирлинга дается в [19], но обозначение $B_{k}$ там относится к $(-1)^{k+1} B_{2 k}$, где $B_{2 k}$ определены, как выше в (13). 


$$
\begin{gathered}
|P(z)| \leqslant K(z) \frac{\left|B_{2}\right|}{2|z|}, \\
\left|P(z)-\sum_{k=1}^{n} \frac{p_{2 k-2}}{z^{2 k-1}}\right| \leqslant K(z) \frac{\left|B_{2(n+1)}\right|}{(2 n+2)(2 n+1)|z|^{2 n+1}}, \quad n=1,2, \ldots,
\end{gathered}
$$

где

$$
K(z)=\max _{u \geqslant 0}\left|\frac{z^{2}}{u^{2}+z^{2}}\right|
$$

(см., например, формулу 6.1.42 в [1]).

Формула 23.1.15 из [1] дает, в частности, асимптотическое поведение чисел Бернулли

$$
B_{2 n+2}=(-1)^{n+2} \frac{2(2 n+2) !}{(2 \pi)^{2 n+2}}(1+o(1)), \quad k \rightarrow \infty,
$$

и, следовательно, оценки (15) оказываются такими же, как в (1), и при этом $a=2 \pi$. Таким образом, ряд Стирлинга (14) служит разложением Жевре для функции $P(z)$ в секторе $S(-\pi / 2, \pi / 2)$.

ЗАмЕчАниЕ 2. Польза примера Стирлинга для нашей темы становится ясной, если принять во внимание следующие обстоятельства:

(i) С учетом формулы (16) оценки (15) гарантируют единственность в секторе $S(-\pi / 2, \pi / 2)$ раствора, равного $\pi$, несмотря на то, что $K(z)$ на границе этого сектора обращается в бесконечность. Так как функция $K(z)$ в неравенстве $(15)$ может быть переписана в виде

$$
K(z)= \begin{cases}1, & -\pi / 4<\arg z<\pi / 4, \\ 1 / \sin (2|\arg z|) & \pi / 4 \leqslant|\arg z|<\pi / 2,\end{cases}
$$

то оказывается, что функция Бине (см. формулу (10)) удовлетворяет всем условиям теоремы 1 с $M(\delta)=1 / \delta$.

(ii) Подставляя в неравенство (15) вместо $n$ значение ${ }^{1)} n_{\text {орt }}(|z|)=[\pi|z|-1]$, получаем

$$
\left|P(z)-\sum_{k=1}^{[\pi|z|-1]} \frac{p_{2 k-1}}{z^{2 k-1}}\right| \leqslant K(z) \frac{2 \sqrt{2 \pi|z|}}{2 \pi|z|-1} e^{-2 \pi|z|} .
$$

Для заданного фиксированного $z \in S(-\pi / 4, \pi / 4)$ оценка (18) указывает наилучшую возможную точность при замене функции $P(z)$ оптимальной конечной суммой ряда Стирлинга, и, как следует из формулы (17), для $|z|>1$ ошибка оказывается меньшей, чем

$$
M e^{-a|z|},
$$

где $M=0,94891$ и $a=2 \pi$.

(iii) Пользуясь равенствами (11), (12), можно также показать, что формальный степенной ряд $\widehat{P}(z)$ служит разложением Жевре для функции $P(z)$ во всем секторе $S(-\pi, \pi)$. Однако оценка (1) с $a=2 \pi$ справедлива лишь для внутреннего подсектора $S(-\pi / 2, \pi / 2)$. Можно показать, что в большем подсекторе

1) Для заданного значения $|z|$ такое значение $n=n_{\mathrm{opt}}(|z|)$ получается как результат минимизации по $n$ выражения в правой части соотношения (15). 
$S(-\pi / 2-\varepsilon, \pi / 2+\varepsilon)$ имеет место оценка, подобная той, что указана в $(1)$, но с постоянной $a$, зависящей от $\varepsilon, a(\varepsilon)=2 \pi \cos \varepsilon$.

В принципе, достаточные условия единственности можно получить, следуя, например, технике, основанной на теореме искажения Альфорса [2] и ее дополнении и уточнении, полученном Варшавским [16]. Однако мы демонстрируем доказательство, которое, во-первых, основано на более простых соображениях и, во-вторых, дает возможность более точно выявить роль каждого из условий (6) и $(7)$.

\section{§5. Основная лемма}

Теорема 1 выводится из следующего обобщения леммы 1.

ЛЕмма 2. Предположим, что функиия $P(z)$ голоморфна в $S(-\pi / 2, \pi / 2)$. Предположим, далее, что для каждого $\delta, 0<\delta<\pi / 2, u$ для всех $z, z \in$ $S(-\pi / 2+\delta, \pi / 2-\delta)$, функиия $P(z)$ имеет оценку

$$
|P(z)| \leqslant M(\delta) e^{-a(\delta)|z|},
$$

где $M(\delta), \log M(\delta)>1$, и а $(\delta), \delta \in(0, \pi / 2)$, удовлетворяют условиям (6) и (7). Тогда

$$
P(z) \equiv 0 .
$$

ДокАЗАТЕЛЬСТво. К сожалению, мы не имеем возможности рассматривать интеграл от функции $e^{z t} P(z)$ ни вдоль мнимой оси, ни вдоль параллельной ей оси. Такое препятствие вполне типично для подобных ситуаций, и мы покажем путь, который иногда дает возможность обойти его.

Задавая $\theta,-\pi / 2<\theta<\pi / 2$, введем в рассмотрение преобразование Лапласа

$$
F_{\theta}(t)=\int_{l_{\theta}} e^{z t} P(z) d z,
$$

где $l_{\theta}=\{z: \arg z=\theta\}$.

В дальнейшем мы покажем, что

(i) функция $F_{\theta}(t)$ может быть аналитически продолжена на всю комплексную плоскость как целая функция $F(t)$, не зависящая от $\theta$;

(ii) функция $F(t)$ ограничена вне любого сектора $S_{\delta}, 0<\delta<\pi / 2$, раствора $2 \delta$ с вершиной в точке $a(\delta) /(2 \sin \delta)$, содержащего интервал $(a(\delta) /(2 \sin \delta),+\infty)$ и симметричного относительно вещественной оси;

(iii) на границе $\partial S_{\delta}$ сектора $S_{\delta}$ выполняется неравенство $|F(t)| \leqslant 2 M(\delta) / a(\delta)$;

(iv) функция $F(t)$ удовлетворяет всем условиям теоремы Карлемана, которую мы приводим ниже и применение которой влечет за собой тождество $F(t) \equiv 0$.

ЗАмЕчАниЕ 3. В. П. Хавин, которому мы показали наш результат, обратил наше внимание на следующее обстоятельство. Если предположить, что функция $M(\delta)$ в $(20)$ подчинена более сильному условию $(9)$ и что функция $a(\delta)$ не зависит от $\delta, a(\delta)=a$, то для любого $c, 0<c<a$, можно указать $h>0$ и $M_{h}>0$, такие, что

$$
|P(h+i y)|<M_{h} e^{-c|y|} .
$$

Тогда, согласно лемме $1, P(z) \equiv 0$. 
Для доказательства неравенства (22) нужно лишь заметить, что если $z=$ $x+i y$ и $x /|y|=\operatorname{tg} \delta$, то с учетом (9) неравенство (20) может быть переписано в виде

$$
|P(z)|<M \exp \left(\frac{b}{\operatorname{arctg}(x /|y|)}\right) e^{-a|z|} .
$$

Далее, если $|y| \gg x>0$, то (23) влечет за собой неравенство

$$
|P(z)|<M^{\prime} \exp \left(\frac{b|y|}{x}\right) e^{-a|y|},
$$

справедливое при некотором выборе постоянной $M^{\prime}>0$. Из этого неравенства при подходящем выборе $x=h$, например $h=b /(a-c)$, немедленно следует $(22)$.

$\mathrm{K}$ сожалению, это простое соображение перестает работать при более быстром росте функции $M(\delta)$, например, таком, как в условии (8).

Итак, мы возвращаемся к доказательству.

5.1. Область аналитичности функции $\boldsymbol{F}_{\boldsymbol{\theta}}(\boldsymbol{t})$. Всюду в доказательстве мы будем пользоваться символом $a$ для обозначения функции $a(\delta)$, за исключением тех случаев, когда нам важно подчеркнуть зависимость этой функции от $\delta$. Предположим также, не ограничивая общности, что $a(\delta)$ является убывающей функцией от $\delta$. Пусть

$$
\delta= \begin{cases}\pi / 2-\theta, & \theta>0 \\ \pi / 2+\theta, & \theta<0 .\end{cases}
$$

Тогда из (20) следует оценка

$$
\left|F_{\theta}(t)\right| \leqslant M(\delta) \int_{l_{\theta}}\left|e^{z t}\right| e^{-a|z|} d|z| .
$$

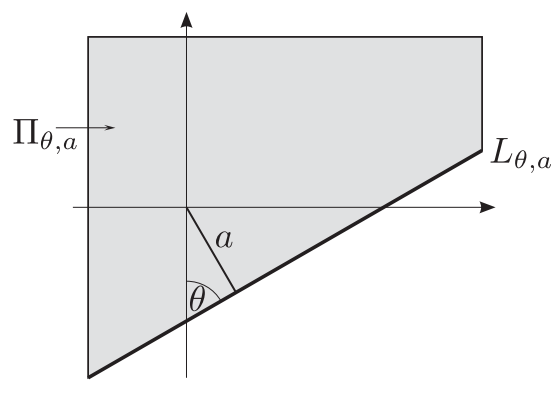

Рис. 1. Прямая $L_{\theta, a}$ и полуплоскость $\prod_{\theta, a}$

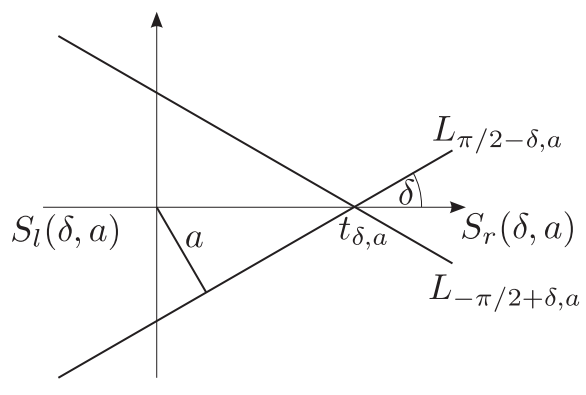

Рис. 2. Прямые $L_{\mp \pi / 2 \pm \delta, a}$ и секторы $S_{l}(\delta, a)$ и $S_{r}(\delta, a)$

Поскольку

$$
\left|e^{z t}\right|=e^{|z|(\sigma \cos \theta-\tau \sin \theta)}, \quad t=\sigma+i \tau,
$$

интеграл (21) абсолютно сходится в полуплоскости вида

$$
\Pi_{\theta, a}=\{t \in \mathbb{C}: \sigma \cos \theta-\tau \sin \theta<a\},
$$


и, значит, функция $F_{\theta}(t)$ голоморфна в этой полуплоскости. В действительности при условии, что, например, $0<\theta<\pi / 2$, прямая

$$
L_{\theta, a}=\{t \in \mathbb{C}: \sigma \cos \theta-\tau \sin \theta=a\}
$$

делит $t$-плоскость на две полуплоскости и $\Pi_{\theta, a}$ является той из них, которая содержит начало координат, см. рис. 1.

Кроме того, из (24) и (25) для $t \in \Pi_{\theta, a}$ следует оценка

$$
\left|F_{\theta}(t)\right| \leqslant M(\delta) \frac{1}{a-(\sigma \cos \theta-\tau \sin \theta)} .
$$

Мы покажем далее, что все функции вида $F_{\theta}(t),|\theta|<\pi / 2$, на самом деле являются элементами единой функции $F(t)$, которая не зависит от $\theta$.

5.2. Соотношение, связывающее $\boldsymbol{F}_{\boldsymbol{\theta}}(\boldsymbol{t})$ и $\boldsymbol{F}_{-\boldsymbol{\theta}}(\boldsymbol{t})$. Задав $\delta, 0<\delta<\pi / 2$, рассмотрим пару функций $F_{\pi / 2-\delta}(t)$ и $F_{-\pi / 2+\delta}(t)$. Согласно сказанному выше, они голоморфны в симметричных относительно вещественной оси полуплоскостях $\Pi_{\pi / 2-\delta, a}$ и $\Pi_{-\pi / 2+\delta, a}$ соответственно,

$$
\begin{aligned}
\Pi_{\pi / 2-\delta, a} & =\{t \in \mathbb{C}: \sigma \sin \delta-\tau \cos \delta<a\}, \\
\Pi_{-\pi / 2+\delta, a} & =\{t \in \mathbb{C}: \sigma \sin \delta+\tau \cos \delta<a\} .
\end{aligned}
$$

На рис. 2 показаны две прямые линии $L_{\pi / 2-\delta, a}$ и $L_{-\pi / 2+\delta, a}$, пересекающиеся в точке

$$
t_{\delta, a}=\frac{a(\delta)}{\sin \delta}
$$

вещественной оси $t$-плоскости под углами к этой оси, равными $\delta$ и $-\delta$ соответственно. Эти прямые определяют два сектора, $S_{l}(\delta, a)$ (левосторонний сектор) и $S_{r}(\delta, a)$ (правосторонний сектор), с вершинами в одной и той же точке $t_{\delta, a}$ и с одним и тем же раствором, равным $2 \delta$.

Введем в рассмотрение секторы

$$
S_{1}(\delta, a)=\Pi_{\pi / 2-\delta, a} \cap \Pi_{-\pi / 2+\delta, a} \quad \text { и } \quad S_{2}(\delta, a)=\Pi_{\pi / 2-\delta, a} \cup \Pi_{-\pi / 2+\delta, a} .
$$

Левосторонний сектор $S_{l}(\delta, a)$ совпадает с $S_{1}(\delta, a)$, а замыкание правостороннего сектора $S_{r}(\delta, a)$ совпадает с дополнением к сектору $S_{2}(\delta, a)$. (См. рис. 3 и рис. 4.)

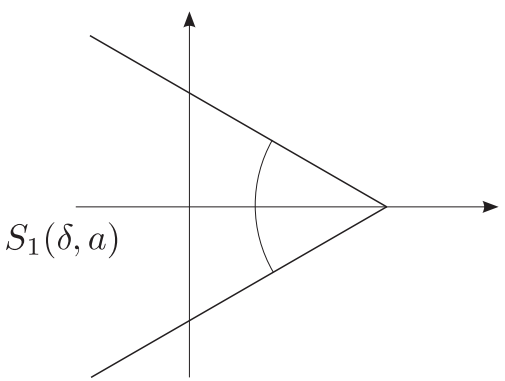

Рис. 3. Сектор $S_{1}(\delta, a)$

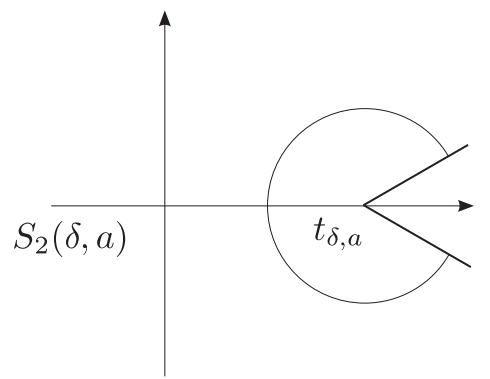

Рис. 4. Сектор $S_{2}(\delta, a)$ 
Пользуясь представлением (21) и теоремой Коши, имеем

$$
F_{\pi / 2-\delta}(t)=F_{-\pi / 2+\delta}(t), \quad t \in S_{1}(\delta, a) .
$$

Таким образом, $F_{\pi / 2-\delta}(t)$ и $F_{-\pi / 2+\delta}(t)$ действительно являются элементами некоторой единой функции $F(t, \delta)$, где

$$
F(t, \delta)= \begin{cases}F_{\pi / 2-\delta}(t), & t \in \Pi_{\pi / 2-\delta, a}, \\ F_{-\pi / 2+\delta}(t), & t \in \Pi_{-\pi / 2+\delta, a},\end{cases}
$$

и эта функция голоморфна в секторе $S_{2}(\delta, a)$.

5.3. Максимальная область аналитичности. Нам понадобится следующая лемма.

ЛЕмма 3. Функиии $F(t, \delta)$ вида (30) являются элементами единой аналитической функиии $F(t)$, которая не зависит от $\delta$ и продолжается на всю комплексную $t$-плоскость как иелая функиия.

ДокАЗАТЕЛЬСТво. Задавая $\delta^{\prime}>\delta^{\prime \prime}>0$, рассмотрим соответствующие функции $F\left(t, \delta^{\prime}\right)$ и $F\left(t, \delta^{\prime \prime}\right)$.

Поскольку функция $a(\delta)$ убывает на интервале $(0, \pi / 2)$, мы имеем

$$
S_{r}\left(\delta^{\prime}, a\right) \supset S_{r}\left(\delta^{\prime \prime}, a\right)
$$

и, следовательно,

$$
\mathbb{C} \backslash S_{r}\left(\delta^{\prime}, a\right) \subset \mathbb{C} \backslash S_{r}\left(\delta^{\prime \prime}, a\right) .
$$

Пользуясь теми же соображениями, что и выше, мы заключаем, что

$$
F\left(t, \delta^{\prime}\right)=F\left(t, \delta^{\prime \prime}\right), \quad t \in \mathbb{C} \backslash S_{r}\left(\delta^{\prime}, a\right),
$$

и, значит, функцию $F\left(t, \delta^{\prime \prime}\right)$ можно рассматривать как аналитическое продолжение функции $F\left(t, \delta^{\prime}\right)$ на большую область $\mathbb{C} \backslash S_{r}\left(\delta^{\prime \prime}, a\right)$.

Выражение (29) для $t_{\delta, a}$ позволяет воспользоваться условием $(7)$ теоремы 1 , которое показывает, что $t_{\delta, a} \rightarrow \infty$ при $\delta \rightarrow 0$, и откуда следует, что

$$
\bigcap_{0<\delta<\pi / 2} S_{r}\left(\delta^{\prime}, a\right)=\varnothing \text {. }
$$

Таким образом, все функции $F(t, \delta), 0<\delta<\pi / 2$, являются элементами единой целой функции $F(t)$, и утверждение (i), тем самым, проверено.

5.4. Оценки для функции $\boldsymbol{F}(\boldsymbol{t})$ в $\boldsymbol{t}$-плоскости. Пользуясь оценкой $(28)$, справедливой в области

$$
\bar{\Pi}_{\theta, a / 2}=\Pi_{\theta, a / 2} \cup L_{\theta, a / 2},
$$

где $\Pi_{\theta, a / 2}$ и $L_{\theta, a / 2}$ определены формулами (26) и (27) соответственно и где $a$ заменено на $a / 2$, мы находим, что

$$
\left|F_{\theta}(t)\right| \leqslant \frac{2 M(\delta)}{a}, \quad t \in \bar{\Pi}_{\theta, a / 2} .
$$

Отсюда и из представления (30) следует, что

$$
|F(t, \delta)| \leqslant \frac{2 M(\delta)}{a}, \quad t \in S_{2}(\delta, a / 2),
$$

и та же оценка справедлива для функции $F(t)$ из п. (i). 
Итак, в секторе $S_{2}(\delta, a / 2)$ функция $F(t, \delta)$, а значит, и функция $F(t)$ являются ограниченными, и (ii) проверено. Кроме того, как следует из (31), функция $F(t)$ удовлетворяет оценке

$$
|F(t)| \leqslant \frac{2 M(\delta)}{a(\delta)}
$$

в секторе $S_{2}(\delta, a / 2)$ и, следовательно, на границе этого сектора. Тем самым утверждение (iii) доказано.

Пользуясь снова условием (7), мы выводим из условия (32) следующую оценкy:

$$
\left|F\left(|t| e^{ \pm i \delta}\right)\right|<\frac{2 M(\delta)}{\delta}
$$

которая справедлива для достаточно малого $\delta$ и которая показывает, что мы находимся в ситуации, когда выполняются условия теоремы Карлемана.

5.5. Теорема Карлемана (см. [4]). Предположим, что $M(\varphi)$ является положительной функиией (конечной или бесконечной) на интервале $(0,2 \pi)$, такой, что $\log M(\varphi)>1$ и интеграл

$$
\int_{0}^{2 \pi} \log \log M(\varphi) d \varphi
$$

конечен. Тогда всякая целая функиия $f(z)$, удовлетворяющая неравенству

$$
|f(z)|<M(\varphi), \quad \varphi=\arg z, 0<\varphi<2 \pi,
$$

тождественна постоянной ${ }^{1)}$.

Таким образом, $F(t)=C$ для некоторой постоянной $C$. Для завершения доказательства п. (iv) остается заметить, что поскольку для каждого $\delta$ справедливо соотношение

$$
F(t, \delta) \rightarrow 0 \text { при } t \rightarrow-\infty,
$$

такое же соотношение выполняется для $F(t)$ и, значит, $F(t) \equiv 0$. Таким образом, п. (iv) доказан, откуда следует, что $P(z) \equiv 0$.

ЗАмечАние 4. Результат Карлемана был в дальнейшем развит Левинсоном и Сьебергом (подробности можно найти, например, в обзоре Содина [14]), получившими максимально общий результат, из которого, в частности, следует распространение теоремы Карлемана на случай, когда сектор заменяется полуполосой. Заметим, однако, что в отличие от этого обобщения теорема Карлемана не нуждается ни в каких дополнительных условиях регулярности мажоранты $M$. Мы полагаем, что это обобщение может быть полезно при исследовании проблемы восстановления, которую мы обсуждаем ниже.

Теорема единственности Ватсона указывает на возможность восстановления функции $P(z)$, удовлетворяющей условиям (i) и (ii) этой теоремы, по коэффициентам формального степенного ряда $\widehat{P}(z)$. Эту задачу можно решить, пользуясь методом суммирования по Борелю. Соответствующий результат мы называем

1)Если функция $M(\varphi)$ удовлетворяет более строгому условию (8), то это заключение немедленно следует из теоремы Фрагмена-Линделёфа. 
теоремой восстановления Ватсона. Доказательство этой теоремы было приведено Ватсоном в цитированной статье [17, Sec. 9]. См. также [18, p. 68, formulae (20)-(22)] и [8, Sec. 8.11].

\section{§6. Теоремы восстановления}

ТЕОРема ВОССТАНОВЛЕНИЯ ВАТСОНА. Предположим, что при некотором $\varepsilon, 0<\varepsilon<\pi / 2$, в секторе $S(-\pi / 2-\varepsilon, \pi / 2+\varepsilon)$ функиия $P(z)$ удовлетворяет условиям (i) и (ii) теоремы единственности Ватсона. Введем в рассмотрение степенной ряд

$$
\widehat{F}(t)=\sum_{n=0}^{\infty} \frac{p_{n}}{n !} t^{n},
$$

который получается как результат суммирования по Борелю формального степенного ряда $\widehat{P}(z)$. Тогда

(i) ряд (33) абсолютно сходится в круге $D_{a}$ комплексной $t$-плоскости радиуса а и с иентром в начале координат и представляет голоморфную в этом круге функиию $F(t)$;

(ii) функиия $F(t)$ допускает аналитическое продолжение из круга $D_{a}$ на область

$$
D_{a, \varepsilon}=D_{a} \cup\{t \in \mathbb{C}:|\arg t|<\varepsilon\},
$$

cм. puc. 5

(iii) функция $P(z)$ может быть представлена в виде преобразования Лапласа функиии $F(t)$,

$$
P(z)=\int_{0}^{+\infty} F(t) e^{-z t} d t
$$

и интеграл абсолютно сходится, когда $z \in S(-\pi / 2, \pi / 2)$.

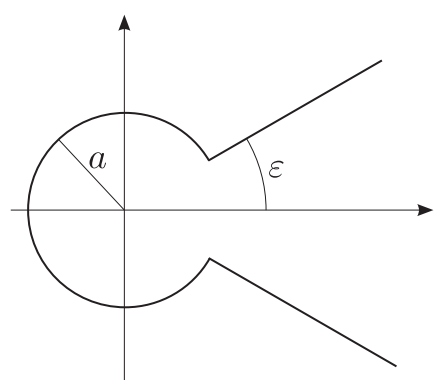

Рис. 5. Область $D_{a, \varepsilon}$

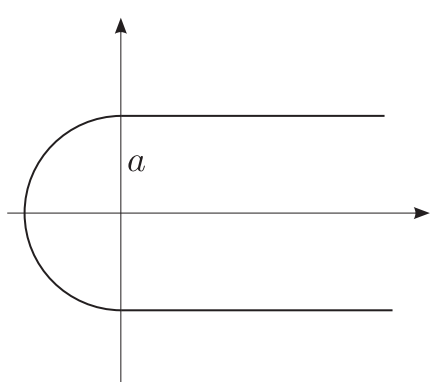

Рис. 6. Область $\mathscr{D}_{a}$

Немедленно возникают вопросы. (а) Что случится с областью аналитичности (34) функции $F(t)$, если устремить $\varepsilon$ к нулю? (b) Предполагая, что в секторе $S(-\pi / 2-\varepsilon, \pi / 2+\varepsilon)$ функция $P(z)$ удовлетворяет условию (1) при фиксированных $a$ и $\varepsilon$, можно ли улучшить результат п. (ii) теоремы восстановления Ватсона?

Ответ на вопрос (а) был указан Ф. Неванлинной в 1919 г., см. [9]. В действительности полученный Неванлинной результат относился к более общей 
ситуации разложений Жевре произвольного порядка $k$, но принципиальная трудность заключается именно в вопросе (а). Любопытно, что Харди в своей монографии по расходящимся рядам, см. [8], обсуждая результаты Ватсона и Неванлинны, не обратил внимание на то улучшение результата Ватсона, которое было указано в [9] для полуплоскости.

Соответствующая же модификация теоремы восстановления Ватсона (ответ на вопрос (b)) появилась лишь 60 лет спустя. Это улучшение заключительной части теоремы восстановления Ватсона было найдено в 1980 г. Аланом Сокалом, который применил свой результат к разложениям в $\phi_{2}^{4}$-квантовой теории поля, см. [15]. Усовершенствование, найденное Сокалом, позволяет указать полную характеризацию для большого класса функций, полученных как результат суммирования по Борелю.

Однако первое обращение к забытой теореме восстановления Ватсона случилось десятью годами раньше в связи с теорией ангармонического осциллятора, см. [7].

Наша теорема 1 подсказывает аналогичный результат для функции $P(z)$, удовлетворяющей условиям теоремы 1 в критическом секторе $S(-\pi / 2, \pi / 2)$, причем для функций $M(\delta)$ и $a(\delta)$ выполняются условия $(6)$ и $(7)$ соответственHO.

Рассмотрим простейший случай, когда $M(\delta)$ и $a(\delta)$ - положительные постоянные. Как было указано ранее, теорема единственности Ватсона распространяется на этот случай, и это дает основание предполагать, что справедлива соответствующая теорема восстановления для $P(z)$.

Введем в рассмотрение область в комплексной плоскости $\mathbb{C}$ вида

$$
\mathscr{D}_{a}=\{t \in \mathbb{C}: \operatorname{dist}(t,(0,+\infty)) \leqslant a\}
$$

(см. рис. 6), которая тем самым полностью определяется положением любой из ее точек. Мы будем называть ее определяющей областью.

ТЕОРЕма воССТАНОВЛЕНИЯ Ф. НЕВАНЛИННЫ. Предположим, что бункиия $P(z)$ удовлетворяет условиям (i) и (ii) теоремы единственности Bатсона в правой полуплоскости z-плоскости. Тогда функиия $F(t)$ вида (33) может быть аналитически продолжена из круга $D_{a}$ на область $\mathscr{D}_{а}$ комплексной t-плоскости как ограниченная функиия; при этом функция $P(z)$ представляется как преобразование Лапласа функиии $F(t)$ из формуль (35), и интеграл абсолютно сходится для $z \in S(-\pi / 2, \pi / 2)$.

Сокал [15] формулирует (без доказательства) следующее обращение теоремы Неванлинны ${ }^{1)}$.

ТеоремА. Задавая $a, a>0$, предположим, что функиия $F(t)$ является аналитической и ограниченной в области $\mathscr{D}_{a}$ комплексной $t$-плоскости $u$ ее ряд Тейлора имеет вид $\sum_{k=0}^{\infty} f_{k} t^{k}$. Определим функиию $P(z)$ как преобразование Лапласа функиии $F(t)$. Тогда она голоморфна в правой полуплоскости z-плоскости и ряд $\sum_{k=0}^{\infty} f_{k} k ! / z^{k+1}$ является ее асимптотическим рядом, удовлетворяющим оценкам (1).

1) В действительности Сокал приводит формально более общий результат, рассматривая наряду с правой полуплоскостью любую полуплоскость вида $\operatorname{Re} z>b$, где $b-$ вещественное число. 
Кроме того, Сокал показал, как, пользуясь теоремой Неванлинны, можно модифицировать утверждение теоремы восстановления Ватсона, и это доказательство вполне простое. В самом деле, предположим, что выполняются условия теоремы восстановления Ватсона, и применим теорему Неванлинны к каждому подсектору сектора $S(-\pi / 2-\varepsilon, \pi / 2+\varepsilon)$ раствора, равного $\pi$. Тогда получается, что функция $F(t)$ вида $(35)$ является голоморфной в области $\mathscr{D}_{a, \theta}=e^{-i \theta} \mathscr{D}_{a}$, $|\theta|<\varepsilon$, для каждого такого $\theta, \theta \in(-\varepsilon, \varepsilon)$, а значит, $F(t)$ голоморфна и в объединении этих областей

$$
\mathscr{D}_{a}(\varepsilon)=\bigcup \mathscr{D}_{a, \theta}
$$

Область (36) ограничена двумя касательными к окружности $\partial D_{a}$, образующими с положительной осью $t$-плоскости углы, равные $\pm \varepsilon$, а также внешней дугой окружности, их соединяющей. (См. рис. 7.)

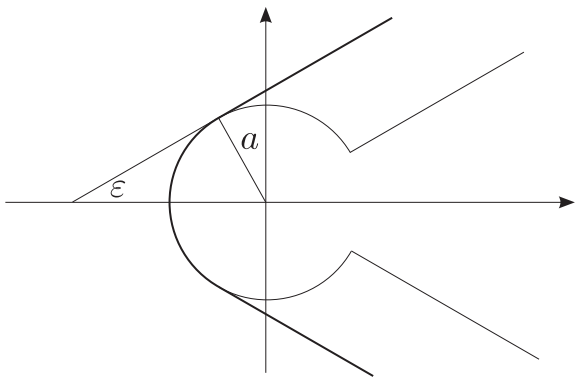

Рис. 7. Область $\mathscr{D}_{a}(\varepsilon)$

ЗАмечАниЕ 5. Попытаемся применить теорему Сокала к ситуации §4. Заметим, что для функции $F(t)$ вида $(12)$ область $\mathscr{D}_{2 \pi}$ является определяющей областью. Поскольку $F(t)$ имеет полюс в точке $t=2 \pi i$, принадлежащей границе области $\mathscr{D}_{2 \pi}$, то она не удовлетворяет условиям этой теоремы. Таким образом, возникает задача такого усовершенствования результата Сокала, которое позволило бы применять ее к неограниченным функциям. Для примера Стирлинга это препятствие может быть немедленно преодолено следующим образом. Введем в рассмотрение функции $F_{0}(t)=1 /\left(t^{2}+4 \pi^{2}\right)$ и $F_{1}(t)=F(t)-F_{0}(t)$. Определяющая область для функции $F_{1}(t)$ имеет вид $\mathscr{D}_{4 \pi}$. Поэтому мы можем, применяя результат Сокала для функции $F_{1}(t)$ и области $\mathscr{D}_{2 \pi}$, получить хорошую оценку для соответствующей функции $P_{1}(z)$ в секторе раствора, большего, чем $\pi$, см. замечание 2(iii). Таким образом, оценка (17) в этом замечании порождается элементарной функцией $F_{0}(t)$.

Мы намерены распространить эти результаты на более общие классы единственности, для которых функция $M(\delta)$ неограниченна. Мы предполагаем, что если заменить условие (6) более сильным условием

$$
M(\delta)<M \exp (b / \delta),
$$

то все еще сохраняется возможность восстановления функции $P(z)$, удовлетворяющей условиям теоремы 1 , по коэффициентам ее разложения Жевре, если использовать при этом, как и выше, метод суммирования по Борелю. 
Для классов функций, удовлетворяющих условиям теоремы 1 , в тех случаях, когда $M(\delta)$ растет быстрее, чем указано в (9), следует, по-видимому, искать альтернативные методы суммирования.

Дальнейшее обсуждение и распространение результатов Неванлинны и Сокала будет приведено в [6].

\section{§7. Заключение}

Мотивацией для настоящей статьи явились следующие два момента. Вопервых, желание понять результат Рамиса и Сибуйа, см. [12], [13], которые показали, что если формальный степенной ряд удовлетворяет аналитическому (не обязательно линейному) дифференциальному уравнению, то, грубо говоря, существует такое $k>0$, что в каждом секторе (критического) раствора $\pi / k$ существует регулярное решение уравнения, для которого указанное формальное является разложением Жевре порядка $k$. Кроме того, они доказали единственность такого решения, и это обстоятельство послужило причиной более внимательного рассмотрения ситуации с распространением теоремы единственности Ватсона на критический сектор. Если $P(z)$ является регулярным решением из теоремы Рамиса и Сибуйа, то мы предполагаем, что $M(\delta)$ удовлетворяет условию (8) и, вероятно, более сильному условию (9). Тем не менее пока это открытый вопрос. Во-вторых, желание найти наилучшую возможную оценку для отклонения функции от конечных сумм ее разложения Жевре. Это может быть сделано, если указать возможно более точные оценки для функций $M(\delta)$ и $a^{-1}(\delta)$, подобные приведенным в (19) для примера Стирлинга.

Благодарности. Авторы благодарны Нику Гарнхаму и Питеру Джонсу и Школе математических наук Свинбурнского технологического университета за их неизменную поддержку, а также Виктору Кацнельсону и Давиду Луси за многочисленные полезные обсуждения. Особую благодарность авторы приносят И. В. Островскому, чьи замечания и предложения позволили существенно улучшить первоначальный вариант статьи.

Второй автор выражает признательность Институту Вейцмана, Израиль, и фонду Рози и Макса Варонов (the Rosi and Max Varon Visiting Professorship program), поддержка которого содействовала завершению этой работы.

\section{ЛитеРАТУРА}

[1] M. J. Abramowitz, I. A. Stegun, Handbook of Mathematical Functions, with Formulas, Graphs, and Mathematical Tables, Dover Publications, New York, 1965.

[2] L. V. Ahlfors, Conformal Invariants. Topic in Geometric Function Theory, McGrawHill, New York-Düsseldorf-Johannesburg, 1973.

[3] T. Carleman, Les fonctions quasy-analytiques, Gauthier-Villars, Paris, 1926.

[4] T. Carleman, "Extension d'un théorème de Liouville", Acta Math., 48 (1926), 363-366; in: Édition Complète des Articles de Torsten Carleman, Inst. Math. Mittag-Leffler, Malmö, 1960, 271-274.

[5] M. Gevrey, "Sur la nature analytique des solutions des équations aux dérivées partielles", Ann. Sci. Écol. Norm. Sup. (3), 35 (1918), 129-190.

[6] D. W. H. Gillam, V. Gurarii, "On the Watson-Nevanlinna recovering theorem", готовится к печати. 
[7] S. Graffi, V. Grecchi, B. Simon, "Borel summability: Application to the anharmonic oscillator", Phys. Lett., 32B (1970), 631-634.

[8] G. H. Hardy, Divergent Series, Oxford: at the Clarendon Press, 1949.

[9] F. Nevanlinna, "Zur Theorie der Asymptotischen Potenzreihen", Ann. Acad. Sci. Fenn. Ser. A, XII:3 (1919), 1-81.

[10] J.-P. Ramis, "Théorèmes d'indices Gevrey pour les équations différentielles ordinaires", Mem. Amer. Math. Soc., 48:296 (1984).

[11] J.-P. Ramis, "Séries divergentes et théories asymptotiques", Bull. Soc. Math. France, 121 (1993).

[12] J.-P. Ramis, Y. Sibuya, "Hukuhara Domain and Fundamental Existence and Uniqueness Theorems for Asymptotic Solutions of Gevrey Type", Asymptotic Analysis, 2 (1989), 39-94.

[13] J.-P. Ramis, Y. Sibuya, "A new proof of multisummability of formal solutions of non linear meromorphic differential equations", Ann. Inst. Fourier, 44:3 (1994), 811-848.

[14] M. Sodin, "Lars Ahlfors' thesis", in: Lectures in Memory of Lars Ahlfors, Israel Math. Conf. Proc. (Haifa, 1996), vol. 14, Bar-Ilan Univ., Ramat Gan, 2000, 113-134.

[15] A. D. Sokal, "An improvement of Watson's theorem on Borel summability", J. Math. Phys., 21:2 (1980), 261-263.

[16] S. Warschawski, "On conformal mapping of infinite strips", Trans. Amer. Math. Soc., 51 (1942), 280-335.

[17] G. N. Watson, "A theory of asymptotic series", Phil. Trans. Roy. Soc. London, Ser. A, 211 (1912), 279-313.

[18] G. N. Watson, "The transformation of an asymptotic series into a convergent series of inverse factorial", Circ. Math. Palermo Rend., 34 (1912), 41-88.

[19] E. T. Whittaker, G. N. Watson, A Course of Modern Analysis, Cambridge Univ. Press, Cambridge, 1996.

Faculty of Engineering and Industrial Sciences

Swinburne University of Technology

e-mail: dgillam@swin.edu.au

School of Mathematical Sciences

Monash University

e-mail: vladimir.vgurarii@sci.monash.edu.au

Поступило в редакцию 7 апреля 2006 г. 